\title{
Emergence of Quantum-Classical Dynamics in an Open Quantum Environment
}

\author{
Kazutomu Shiokawa and Raymond Kapral \\ Chemical Physics Theory Group, Department of Chemistry, \\ University of Toronto, Toronto, ON M5S 3H6, Canada
}

\begin{abstract}
The conditions under which an open quantum mechanical system may be described by mixed quantum-classical dynamics are investigated. Decoherence is studied using influence functional methods in a model composite quantum system comprising two coupled systems, $A$ and $C$, interacting with a harmonic bath with Ohmic and super-Ohmic spectral densities. Subsystem $A$ is directly coupled to subsystem $C$, while $C$ is coupled directly to the bath. Calculations are presented for a model where subsystem $A$ is taken to be a two-level system which is bilinearly coupled to a single harmonic oscillator $C$ subsystem. The loss of quantum coherence in each subsystem is discussed in the extreme non-adiabatic regime where the dynamics of subsystem $A$ is essentially frozen. Subsystem $C$ is shown to lose its coherence rapidly, while subsystem $A$ maintains coherence for longer time periods since $C$ modulates the influence of the bath on $A$. Thus, one may identify situations where the coupled $A C$ system evolution effectively obeys mixed quantum-classical dynamics.
\end{abstract}

\section{INTRODUCTION}

Since it is often impossible to isolate a quantum system completely from its environment, the study of open quantum dynamical systems is necessary in many circumstances. Techniques used to treat open quantum systems have been developed extensively and used in numerous applications. 3 Projection operator techniquest influence functional methods 9 have been actively used in chemical physics in studies of electron, proton, and exciton transfer processes in the condensed phase and in biological systems. 10

In this paper, we investigate some of the circumstances under which an open quantum system can be described using mixed quantum-classical dynamics. Quantumclassical dynamics is used to study condensed phase many-body systems 11, 12 especially in the context of methods for treating non-adiabatic dynamics 1320 In the studies presented here, we consider a composite quantum mechanical system $A C$ comprising two coupled subsystems $A$ and $C$; subsystem $C$ is assumed to be in direct contact with a thermal quantum mechanical bath $B$. Systems with this structure can arise in condensed phase dynamics where certain quantum degrees of freedom $(A)$, for example, those associated with protons or electrons, may interact directly with neighboring solvent molecules $(C)$, which in turn interact with the rest of the solvent $(B)$. It is interesting to determine if the composite system may be treated as mixed quantum-classical system where the dynamics of subsystem $C$, which is in direct contact with the heat bath, is classical in character while subsystem $A$ retains its quantum nature. Some aspects of the dynamics of such quantum-classical composite systems have been investigated.21

In order to investigate this problem we study a simple model system where subsystem $A$ depends on spin degrees of freedom and subsystem $C$ is a single harmonic oscillator bilinearly coupled both to subsystem $A$ and the bath. The bath is a collection of independent harmonic oscillators. While this is a highly simplified model of the realistic systems discussed above, it does capture some essential features of real coupled systems and is amenable to detailed analysis. Due to its interaction with the bath, the dynamics of subsystem $C$ is dissipative and executes brownian motion. The brownian motion of a quantum particle governed by different potential functions and immersed in a thermal harmonic oscillator bath has been studied extensively by influence functional methods 2227 The character of its dynamics is determined by the system-bath coupling and the spectral density and temperature of the bath. In the composite system we study, the dynamics of the $C$ subsystem oscillator is also influenced by the quantum dynamics of subsystem $A$. The dynamics of subsystem $A$ is more complicated. It is also dissipative but its energy must be transmitted through subsystem $C$ to the bath. Our results on the applicability of mixed quantum-classical dynamics are based on the nature of decoherence28.29 in the coupled system: when one subsystem behaves quantum mechanically and the other classically, there must be a mechanism making the former decohere slowly and the latter quickly.

The outline of this paper is as follows: In Sec. II we specify the model in detail and outline the application of the influence functional formalism to it. Although we use influence functional methods, similar results can be obtained by other methods. Section III] considers the equilibration of the $C B$ system in the absence of $A$ and establishes that this composite system can be described by an effective spectral density. The emergence of mixed quantum-classical dynamics is investigated in Sec. IV. In this section we use the results developed in the previous sections, specialized to a two-level system in the strongly non-adiabatic regime where the populations change very slowly. We study the decoherence of the off-diagonal elements of the subsystem $A$ reduced density matrix in 
this regime and show under what conditions one may observe quantum-classical dynamics. The conclusions of the study are presented in Sec. V. The Appendices contain additional details of the calculations for Ohmic and super-Ohmic spectral densities.

\section{GENERAL FORMULATION}

\section{A. Composite system in a thermal bath}

The composite $A C B$ quantum system consists of three coupled subsystems: subsystem $A$ is coupled directly to subsystem $C$, while subsystem $C$ is in direct contact with a thermal bath $B$. The $A C B$ system hamiltonian is,

$$
\hat{H}=\hat{H}_{A}+\hat{V}_{A C}+\hat{H}_{C}+\hat{V}_{C B}+\Delta \hat{H}_{C}+\hat{H}_{B} .
$$

The thermal bath is taken to be a set of $N$ independent harmonic oscillators with frequencies $\omega_{n}$, masses $m_{n}$, and coordinates and conjugate momenta $(\hat{q}, \hat{p}) \equiv$ $\left(\hat{q}_{1}, \ldots, \hat{q}_{N}, \hat{p}_{1}, \ldots, \hat{p}_{N}\right)$ so that the hamiltonian is

$$
\hat{H}_{B}=\sum_{n=1}^{N}\left(\frac{\hat{p}_{n}^{2}}{2 m_{n}}+\frac{1}{2} m_{n} \omega_{n}^{2} \hat{q}_{n}^{2}\right) .
$$

Subsystem $C$ consists of a single harmonic oscillator with renormalized mass $M$ and frequency $\omega_{r}$ with coordinate and momentum operators $(\hat{x}, \hat{p})$,

$$
\hat{H}_{C}=\frac{\hat{p}^{2}}{2 M}+\frac{M}{2} \omega_{r}^{2} \hat{x}^{2} .
$$

The interaction potential between $C$ and the bath is assumed to be bilinear,

$$
\hat{V}_{C B}=\hat{x} \sum_{n=1}^{N} c_{n} \hat{q}_{n},
$$

where $c_{n}$ is the coupling constant to the $n$th oscillator. The coupling constants are related to the spectral density of the bath by,

$$
J_{B}(\omega) \equiv \pi \sum_{n} \frac{c_{n}^{2}}{2 m_{n} \omega_{n}} \delta\left(\omega-\omega_{n}\right)
$$

We suppose the spectral density has the form

$$
J_{B}(\omega)=\eta \omega^{\nu} e^{-\omega / \Lambda},
$$

and consider both Ohmic $(\nu=1)$ and super-Ohmic $(\nu=3)$ cases. A counter term $\Delta \hat{H}_{C}$ has been added to the hamiltonian. It depends on $c_{n}, m_{n}, \omega_{n}, \hat{p}$ and $\hat{x}$ and is given by

$$
\Delta \hat{H}_{c}= \begin{cases}\eta \Lambda \hat{x}^{2} / \pi & (\nu=1) \\ \eta \Lambda \hat{p}^{2} / M^{2} \pi+\eta \Lambda^{3} \hat{x}^{2} / \pi & (\nu=3) .\end{cases}
$$

This term is introduced to cancel the shift of the mass and frequency of the $C$ subsystem oscillator due to the interaction with the bath which will become divergent when the frequency cutoff $\Lambda$ goes to infinity. As is customary, we consider the renormalized quantities after including a counter term as physical observables with specified values in our analysis 30 The hamiltonian of subsystem $A, \hat{H}_{A}(\sigma)$, depends on spin variables $\sigma$ and we assume subsystem $A$ is bilinearly coupled to subsystem $C, \hat{V}_{A C}=\lambda \sigma \hat{x}$.

The dynamical properties of interest can be computed from the density matrix of the system at time $t$. In the coordinate representation $\{x, q\}$ of the $C$ subsystem and bath $B$, the density matrix takes the form, $\hat{\rho}\left(x, x^{\prime}, q, q^{\prime}, t\right) \equiv\left\langle x q|\hat{\rho}(t)| x^{\prime} q^{\prime}\right\rangle$, which is still an operator in the $A$ subsystem degrees of freedom. The density matrix may be written more explicitly in integral form in terms of the propagator

$$
\hat{K}\left(x, q ; t \mid x_{0}, q_{0} ; 0\right) \equiv\left\langle x q\left|e^{-i \hat{H} t / \hbar}\right| x_{0} q_{0}\right\rangle,
$$

as

$$
\begin{aligned}
\hat{\rho}\left(x, x^{\prime}, q, q^{\prime}, t\right) & =\int d x_{0} d x_{0}^{\prime} d q_{0} d q_{0}^{\prime} \hat{K}\left(x, q ; t \mid x_{0}, q_{0} ; 0\right) \\
& \times \hat{\rho}\left(x_{0}, x_{0}^{\prime}, q_{0}, q_{0}^{\prime}, 0\right) \hat{K}^{*}\left(x^{\prime}, q^{\prime} ; t \mid x_{0}^{\prime}, q_{0}^{\prime} ; 0\right) .
\end{aligned}
$$

We are primarily interested in the dynamics of the composite $A C$ subsystem under the influence of the thermal bath. In such a circumstance the reduced density matrix $\hat{\rho}_{r}$, obtained by integrating over the bath degrees of freedom, is the relevant quantity. Such a reduction is especially appropriate if the characteristic time scale for the bath is much shorter than those for the $A$ and $C$ subsystems. We assume a factorized initial condition between the $A C$ subsystem and the bath,

$\hat{\rho}\left(x_{0}, x_{0}^{\prime}, q_{0}, q_{0}^{\prime}, 0\right)=\hat{\rho}_{A C}\left(x_{0}, x_{0}^{\prime}, 0\right) \otimes \rho_{B}\left(q_{0}, q_{0}^{\prime}, 0\right)$,

and the bath is always taken to be initially in thermal equilibrium. Under these conditions the integral form of the reduced density matrix at time $t$ is

$\hat{\rho}_{r}\left(x, x^{\prime}, t\right)=\int d x_{0} d x_{0}^{\prime} \hat{J}_{r}\left(x, x^{\prime} ; t \mid x_{0}, x_{0}^{\prime} ; 0\right) \hat{\rho}_{A C}\left(x_{0}, x_{0}^{\prime}, 0\right)$,

where the time evolution kernel $J_{r}$ is given by

$$
\begin{aligned}
\hat{J}_{r}\left(x, x^{\prime} ; t \mid x_{0}, x_{0}^{\prime} ; 0\right) & =\int d q d q_{0} d q_{0}^{\prime} \hat{K}\left(x, q ; t \mid x_{0}, q_{0} ; 0\right) \\
& \times \rho_{B}\left(q_{0}, q_{0}^{\prime}, 0\right) \hat{K}^{*}\left(x^{\prime}, q ; t \mid x_{0}^{\prime}, q_{0}^{\prime} ; 0\right) .
\end{aligned}
$$

Given the initial conditions discussed above, this exact expression for the reduced density matrix specifies a nonMarkovian time evolution since the solution at time $t$ depends on its past history. Approximate Markovian evolution equations may miss essential features of the quantum/classical correspondence and tend to underestimate 
the loss of quantum coherence. In addition, a Markovian approximation is not generally valid for a harmonic oscillator model, except for systems with Ohmic type dissipation in the high temperature regime. Below we use influence functional methods where the exact solution is expressed as a path integral in order to deal with the nonlocal time evolution.

\section{B. Influence functional method}

The formulation of the reduced density matrix in terms of an influence functional for the composite $A C B$ system parallels that for spin-boson models often discussed in the literature.3, 31 Consequently the presentation of the generalization to our system will be brief. In order to write the reduced density matrix in terms of an influence functional, we first introduce a basis of spin functions with labels $\sigma$ and $\sigma^{\prime}$ to represent the states of the $A$ subsystem. In this basis we may write eq. (11) as,

$$
\begin{aligned}
& \rho_{r}\left(\sigma, \sigma^{\prime}, x, x^{\prime}, t\right)=\int d \sigma_{0} d \sigma_{0}^{\prime} \int d x_{0} d x_{0}^{\prime} \\
& \times J_{r}\left(\sigma, \sigma^{\prime}, x, x^{\prime} ; t \mid \sigma_{0}, \sigma_{0}^{\prime}, x_{0}, x_{0}^{\prime} ; 0\right) \rho_{r}\left(\sigma_{0}, \sigma_{0}^{\prime}, x_{0}, x_{0}^{\prime}, 0\right) .
\end{aligned}
$$

For the factorized initial condition (10) the kernel $J_{r}$ can be written in the path integral form as

$$
\begin{aligned}
& J_{r}\left(\sigma, \sigma^{\prime}, x, x^{\prime} ; t \mid \sigma_{0}, \sigma_{0}^{\prime}, x_{0}, x_{0}^{\prime} ; 0\right)= \\
& \int_{\left(\sigma_{0} \sigma_{0}^{\prime}\right)}^{\left(\sigma \sigma^{\prime}\right)} \mathcal{D} \sigma \mathcal{D} \sigma^{\prime} K[\sigma] K^{*}\left[\sigma^{\prime}\right] J_{C}^{\sigma \sigma^{\prime}}\left(x, x^{\prime} ; t \mid x_{0}, x_{0}^{\prime} ; 0\right),
\end{aligned}
$$

where $K[\sigma]$ is a probability amplitude for subsystem $A$ following the path $\sigma$ in the absence of the subsystem $C$ and the environment. The evolution kernel $J_{C}^{\sigma \sigma^{\prime}}$ for subsystem $C$ in the presence of external sources $\left(\sigma, \sigma^{\prime}\right)$ is

$$
J_{C}^{\sigma \sigma^{\prime}}\left(x, x^{\prime} ; t \mid x_{0}, x_{0}^{\prime} ; 0\right) \equiv \int_{\left(x_{0} x_{0}^{\prime}\right)}^{\left(x x^{\prime}\right)} \mathcal{D} x \mathcal{D} x^{\prime} e^{i \mathcal{S}\left[\sigma, \sigma^{\prime}, x, x^{\prime}\right] / \hbar}
$$

The action $\mathcal{S}\left[\sigma, \sigma^{\prime}, x, x^{\prime}\right]$ consists of several contributions and can be decomposed as follows:

$$
\begin{aligned}
\mathcal{S}\left[\sigma, \sigma^{\prime}, x, x^{\prime}\right]= & \mathcal{S}_{C}\left[x, x^{\prime}\right]+\mathcal{S}_{A C}\left[\sigma, \sigma^{\prime}, x, x^{\prime}\right] \\
& +\Delta \mathcal{S}_{C}\left[x, x^{\prime}\right]+\mathcal{S}_{I F}\left[x, x^{\prime}\right] .
\end{aligned}
$$

The actions for subsystem $C$, plus its counter action, and the interaction between subsystems $A$ and $C$ are

$$
\begin{aligned}
\left(\mathcal{S}_{C}+\Delta \mathcal{S}_{C}\right)\left[x, x^{\prime}\right]= & \int_{0}^{t} d s\left(\frac{M_{0}}{2} \dot{x}^{2}(s)-\frac{M_{0}}{2} \omega_{0}^{2} x^{2}(s)\right. \\
& \left.-\frac{M_{0}}{2}{\dot{x^{\prime}}}^{2}(s)+\frac{M_{0}}{2} \omega_{0}^{2} x^{\prime 2}(s)\right), \\
\mathcal{S}_{A C}\left[\sigma, \sigma^{\prime}, x, x^{\prime}\right]= & \lambda \int_{0}^{t} d s\left(x(s) \sigma(s)-x^{\prime}(s) \sigma^{\prime}(s)\right),
\end{aligned}
$$

where, for notational convenience, we used the bare mass $M_{0}$ and bare frequency $\omega_{0}$ as $M_{0}=M$ and $M_{0} \omega_{0}^{2}=$ $M \omega_{r}^{2}+2 \eta \Lambda / \pi$ for $\nu=1$ while $M_{0}=M+2 \eta \Lambda / \pi$ and $M_{0} \omega_{0}^{2}=M \omega_{r}^{2}+2 \eta \Lambda^{3} /(3 \pi)$ for $\nu=3$.

The influence action $\mathcal{S}_{I F}\left[x, x^{\prime}\right]$ accounts for the effect of the bath on $C$ and is given by

$$
\begin{aligned}
\mathcal{S}_{I F}\left[x, x^{\prime}\right]= & i \int_{0}^{t} d s \int_{0}^{s} d s^{\prime}\left[x(s)-x^{\prime}(s)\right] \times \\
& {\left[\alpha\left(s-s^{\prime}\right) x\left(s^{\prime}\right)-\alpha^{*}\left(s-s^{\prime}\right) x^{\prime}\left(s^{\prime}\right)\right], }
\end{aligned}
$$

where $\alpha(t)$ is a complex kernel whose real $\alpha^{R}(t)$ and imaginary $\alpha^{I}(t)$ parts, respectively, are given by

$$
\begin{aligned}
\alpha^{R}(t) & =\frac{1}{\pi} \int_{0}^{\infty} d \omega J_{B}(\omega) \operatorname{coth} \frac{\beta \hbar \omega}{2} \cos \omega t \\
\alpha^{I}(t) & =-\frac{1}{\pi} \int_{0}^{\infty} d \omega J_{B}(\omega) \sin \omega t
\end{aligned}
$$

We define new variables as $R \equiv\left(x+x^{\prime}\right) / 2, r \equiv x-x^{\prime}$, $\sigma_{ \pm} \equiv \sigma \pm \sigma^{\prime}$, and write the actions in these new variables as

$$
\begin{gathered}
\mathcal{S}_{A C}\left[\sigma_{ \pm}, R, r\right]=\frac{\lambda}{2} \int_{0}^{t} d s\left(\sigma_{+}(s) r(s)+2 \sigma_{-}(s) R(s)\right) \\
\left(\mathcal{S}_{C}+\Delta \mathcal{S}_{C}\right)[R, r]=\int_{0}^{t} d s\left\{M_{0} \dot{R}(s) \dot{r}(s)-M_{0} \omega_{0}^{2} R(s) r(s)\right\} \\
\mathcal{S}_{I F}[R, r]=i \int_{0}^{t} d s \int_{0}^{s} d s^{\prime} r(s) \alpha^{R}\left(s-s^{\prime}\right) r\left(s^{\prime}\right) \\
-2 \int_{0}^{t} d s \int_{0}^{s} d s^{\prime} r(s) \alpha^{I}\left(s-s^{\prime}\right) R\left(s^{\prime}\right) .
\end{gathered}
$$

\section{Euler-Lagrange equations and solution}

¿From eqs. (21) the Euler-Lagrange equations for $R$ and $r$ are

$$
\begin{aligned}
M_{0} \ddot{R}_{c}(s)+M_{0} \omega_{0}^{2} R_{c}(s) & +2 \int_{0}^{s} d s^{\prime} \alpha^{I}\left(s-s^{\prime}\right) R_{c}\left(s^{\prime}\right) \\
& =\frac{1}{2} \lambda \sigma_{+}(s), \\
M_{0} \ddot{r}_{c}(s)+M_{0} \omega_{0}^{2} r_{c}(s) & -2 \int_{s}^{t} d s^{\prime} \alpha^{I}\left(s-s^{\prime}\right) r_{c}\left(s^{\prime}\right) \\
& =\lambda \sigma_{-}(s) .
\end{aligned}
$$

The initial and final conditions for eq. (22) (eq. 23)) are $R_{0}$ and $R_{t}\left(r_{0}\right.$ and $\left.r_{t}\right)$. If we let the two independent solutions of the homogeneous part of eq. (22) (eq. (23)) be $u_{i}(s)\left(v_{i}(s)\right), i=1,2$, with boundary conditions $u_{1}(0)=1, u_{1}(t)=0, u_{2}(0)=0, u_{2}(t)=1$ 
$\left(v_{1}(0)=1, v_{1}(t)=0, v_{2}(0)=0, v_{2}(t)=1\right)$, the solutions of these uncoupled equations can be written as

$$
\begin{aligned}
R_{c}(s) & =R_{0} u_{1}(s)+R_{t} u_{2}(s)+\frac{\mu}{2} \sigma_{+}(g(s)), \\
r_{c}(s) & =r_{0} v_{1}(s)+r_{t} v_{2}(s)+\mu \sigma_{-}(g(s)),
\end{aligned}
$$

where we have used the notation,

$$
\begin{aligned}
\sigma_{+}(g(s)) & \equiv \int_{0}^{s} d s^{\prime} g_{+}\left(s-s^{\prime}\right) \sigma_{+}\left(s^{\prime}\right) \\
& -u_{2}(s) \int_{0}^{t} d s^{\prime} g_{+}\left(t-s^{\prime}\right) \sigma_{+}\left(s^{\prime}\right) \\
\sigma_{-}(g(s)) & \equiv \int_{0}^{s} d s^{\prime} g_{-}\left(s-s^{\prime}\right) \sigma_{-}\left(s^{\prime}\right) \\
& -v_{2}(s) \int_{0}^{t} d s^{\prime} g_{-}\left(t-s^{\prime}\right) \sigma_{-}\left(s^{\prime}\right) .
\end{aligned}
$$

and defined $\mu \equiv \lambda / M$. The solutions $v_{1}$ and $v_{2}$ satisfy the homogeneous part of the backward time equation (23) and are related to $u_{1}$ and $u_{2}$ by $v_{1}(s)=u_{2}(t-s)$ and $v_{2}(s)=u_{1}(t-s)$. The function $g_{+}(s)\left(g_{-}(s)\right)$ also satisfies the homogeneous part of eq. (22) (eq. (23)) with boundary conditions $g_{ \pm}(0)=0, \dot{g}_{ \pm}(0)=1$.

The solutions for $g_{ \pm}$are given in Appendix A for Ohmic and super-Ohmic spectral densities. From these solutions $u_{1,2}$ and $v_{1,2}$ can be determined.

\section{Reduced density matrix solution}

Since the potentials in our model are harmonic, an exact evaluation of the path integral can be carried out. It is dominated by the classical solution given in eq. (24). The $\sigma$ dependence in eq. (24) arises from the back reaction of the $A$ subsystem on the $C$ subsystem. To separate out the contribution from this back reaction, we expand $\mathcal{S}$ in powers of $\sigma$ and write,

$$
\mathcal{S}\left[\sigma_{ \pm}, R_{c}, r_{c}\right]=\mathcal{S}^{(0)}\left[R_{c}, r_{c}\right]+\mathcal{S}^{(1)}\left[\sigma_{ \pm}, R_{c}, r_{c}\right]+\mathcal{S}^{(2)}\left[\sigma_{ \pm}\right]
$$

The zeroth order term in $\sigma$ takes the form

$$
\begin{aligned}
\mathcal{S}^{(0)}\left[R_{c}, r_{c}\right]= & \left(M \dot{u}_{1}(t) R_{0}+M \dot{u}_{2}(t) R_{t}\right) r_{t} \\
- & \left(M \dot{u}_{1}(0) R_{0}+M \dot{u}_{2}(0) R_{t}\right) r_{0} \\
+ & i\left(a_{11}(t) r_{0}^{2}+\left(a_{12}(t)+a_{21}(t)\right) r_{0} r_{t}\right. \\
& \left.+a_{22}(t) r_{t}^{2}\right)
\end{aligned}
$$

and is an influence action for a damped harmonic oscillator 24, 26 Here

$$
a_{k l}(t)=\frac{1}{2} \int_{0}^{t} d s \int_{0}^{t} d s^{\prime} v_{k}(s) \alpha^{R}\left(s-s^{\prime}\right) v_{l}\left(s^{\prime}\right),
$$

for $(k, l=1,2)$ contains the effects of fluctuations due to the bath on subsystem $C$. The term linear in $\sigma$ is

$$
\begin{aligned}
& \mathcal{S}^{(1)}\left[\sigma_{ \pm}, R_{c}, r_{c}\right]=\lambda \int_{0}^{t} d s\left(u_{1}(s) R_{0}+u_{2}(s) R_{t}\right) \sigma_{-}(s) \\
+ & i \mu r_{0} \int_{0}^{t} d s \int_{0}^{t} d s^{\prime}\left\{v_{1}(s) \alpha^{R}\left(s-s^{\prime}\right) \sigma_{-}\left(g\left(s^{\prime}\right)\right)\right\} \\
+ & i \mu r_{t} \int_{0}^{t} d s \int_{0}^{t} d s^{\prime}\left\{v_{2}(s) \alpha^{R}\left(s-s^{\prime}\right) \sigma_{-}\left(g\left(s^{\prime}\right)\right)\right\} \\
+ & \frac{\lambda r_{0}}{2} \int_{0}^{t} d s v_{1}(s) \sigma_{+}(s)+\frac{\lambda r_{t}}{2} \int_{0}^{t} d s v_{2}(s) \sigma_{+}(s) .
\end{aligned}
$$

This term arises from the interaction between subsystem $A$ and the bath, modulated by the trajectory of subsystem $C$. The quantum back reaction of subsystem $A$ on $C$ induces self-coupling in $A$, which is contained in the last term,

$$
\begin{aligned}
\mathcal{S}^{(2)}\left[\sigma_{ \pm}\right] & =\frac{\lambda \mu}{2} \int_{0}^{t} d s \sigma_{-}(s) \sigma_{+}(g(s)) \\
& +i \mu^{2} \int_{0}^{t} d s \int_{0}^{s} d s^{\prime} \sigma_{-}(g(s)) \alpha^{R}\left(s-s^{\prime}\right) \sigma_{-}\left(g\left(s^{\prime}\right)\right)
\end{aligned}
$$

Using the results above, $J_{C}^{\sigma \sigma^{\prime}}$ in eq. (15) can be written in the compact form,

$$
J_{C}^{\sigma \sigma^{\prime}}\left(R_{t}, r_{t} ; t \mid R_{0}, r_{0} ; 0\right)=N(t) \exp \left\{\frac{i}{\hbar} \mathcal{L}\right\},
$$

where $\mathcal{L}=\vec{R}^{T} \mathbf{u} \vec{r}+i \vec{r}^{T} \mathbf{a} \vec{r}+\vec{\sigma}_{u}^{T} \vec{R}+\vec{\sigma}_{v}^{T} \vec{r}+\mathcal{S}^{(2)}\left[\sigma_{ \pm}\right]$and $N(t)$ is a normalization factor independent of dynamical variables. In writing this equation we have introduced the notation and $(\mathbf{a})_{i j}=a_{i j}, \vec{R}^{T}=\left(R_{0}, R_{t}\right)$ and $\vec{r}^{T}=\left(r_{0}, r_{t}\right)$, where $T$ stands for the transpose. The matrix $\mathbf{u}$ is defined as

$$
\mathbf{u}=\left(\begin{array}{ll}
u_{11} & u_{12} \\
u_{21} & u_{22}
\end{array}\right) \equiv M\left(\begin{array}{cc}
-\dot{u}_{1}(0) & \dot{u}_{1}(t) \\
-\dot{u}_{2}(0) & \dot{u}_{2}(t)
\end{array}\right)
$$

while the vectors $\vec{\sigma}_{u}$ and $\vec{\sigma}_{v}$ are given by

$$
\vec{\sigma}_{u}=\lambda\left(\begin{array}{c}
\sigma_{-}\left(u_{1}\right) \\
\sigma_{-}\left(u_{2}\right)
\end{array}\right), \quad \vec{\sigma}_{v}=\lambda\left(\begin{array}{c}
\sigma\left(v_{1}\right) \\
\sigma\left(v_{2}\right)
\end{array}\right) .
$$

In eqs. (33) we have used the symbolic notation

$$
\begin{aligned}
\sigma_{-}\left(u_{i}\right) \equiv & \int_{0}^{t} d s u_{i}(s) \sigma_{-}(s) \\
\sigma\left(v_{i}\right) \equiv & \int_{0}^{t} d s v_{i}(s)\left[\sigma_{+}(s) / 2\right. \\
& \left.+\int_{0}^{t} d s^{\prime} i \alpha^{R}\left(s-s^{\prime}\right) \sigma_{-}\left(g\left(s^{\prime}\right)\right) / M\right],
\end{aligned}
$$

for $i=1,2$.

Having derived the expression for $J_{C}^{\sigma \sigma^{\prime}}$ in eq. (31), we may use it to obtain the partial Wigner transform of the 
reduced density matrix The density matrix in the partial Wigner representation 32 over the $C$ subsystem degrees of freedom is defined by

$$
\begin{aligned}
\rho_{r W}\left(\sigma, \sigma^{\prime}, R, P, t\right)= & \frac{1}{2 \pi \hbar} \int d r e^{-i P r / \hbar} \\
& \times \rho_{r}\left(\sigma, \sigma^{\prime}, R+r / 2, R-r / 2, t\right) .
\end{aligned}
$$

Wigner transforming eq. (13) and using this definition we obtain

$$
\begin{aligned}
& \rho_{r W}\left(\sigma, \sigma^{\prime}, R, P, t\right)=\int d \sigma_{0} d \sigma_{0}^{\prime} \int_{\left(\sigma_{0} \sigma_{0}^{\prime}\right)}^{\left(\sigma \sigma^{\prime}\right)} \mathcal{D} \sigma \mathcal{D} \sigma^{\prime} K[\sigma] K^{*}\left[\sigma^{\prime}\right] \\
& \int d R_{0} d P_{0} K_{C}^{\sigma \sigma^{\prime}}\left(R, P ; t \mid R_{0}, P_{0} ; 0\right) \\
& \left.\times \rho_{r W(} \sigma_{0}, \sigma_{0}^{\prime}, R_{0}, P_{0}, 0\right),
\end{aligned}
$$

where the kernel $K_{C}^{\sigma \sigma^{\prime}}\left(R, P ; t \mid R_{0}, P_{0} ; 0\right)$ is defined by

$$
\begin{aligned}
K_{C}^{\sigma \sigma^{\prime}}\left(R, P ; t \mid R_{0}, P_{0} ; 0\right)= & \frac{1}{2 \pi \hbar} \int d r d r_{0} e^{-i\left(P r-P_{0} r_{0}\right) / \hbar} \\
& \times J_{C}^{\sigma \sigma^{\prime}}\left(R, r ; t \mid R_{0}, r_{0} ; 0\right) .
\end{aligned}
$$

Inserting the expression for $J_{C}^{\sigma \sigma^{\prime}}$ in eq. (31), the time evolution kernel $K_{C}^{\sigma \sigma^{\prime}}$ for the Wigner function is given by

$$
\begin{aligned}
K_{C}^{\sigma \sigma^{\prime}}\left(R_{t}, P_{t} ; t \mid\right. & \left.R_{0}, P_{0} ; 0\right) \\
= & \frac{N(t)}{2 \pi \hbar} \int d r d r_{0} e^{i\left(-P_{t} r_{t}+P_{0} r_{0}+\mathcal{L}\right) / \hbar} \\
= & N_{W}(t) \exp \left[-\delta \vec{X}_{W}^{T} \mathbf{Q}^{-1} \delta \vec{X}_{W}\right. \\
& \left.+\frac{i}{\hbar} \vec{\sigma}_{u}^{T} \vec{R}+\frac{i}{\hbar} \mathcal{S}^{(2)}\left[\sigma_{ \pm}\right]\right],
\end{aligned}
$$

where $N_{W}(t)=N(t) / 2 \hbar \sqrt{|\mathbf{a}|}$ and $|\mathbf{a}|$ is the determinant of a. The vector $\delta \vec{X}_{W}=\vec{X}_{W}-\langle\vec{X}\rangle$, with

$$
\vec{X}_{W}=\left(\begin{array}{c}
R_{t} \\
P_{t}-\lambda \sigma\left(v_{2}\right)
\end{array}\right)
$$

and

$$
\langle\vec{X}\rangle=\left(\begin{array}{c}
\langle R\rangle \\
\langle P\rangle
\end{array}\right)=\frac{-1}{u_{21}}\left(\begin{array}{cc}
u_{11} & 1 \\
|\mathbf{u}| & u_{22}
\end{array}\right)\left(\begin{array}{c}
R_{0} \\
P_{0}+\lambda \sigma\left(v_{1}\right)
\end{array}\right) .
$$

The matrix $\mathbf{Q}$ is given by

$$
\begin{aligned}
& \mathbf{Q}=\frac{4 \hbar}{u_{21}^{2}} \times \\
& \left(\begin{array}{cc}
a_{11} & a_{12} u_{21}-a_{11} u_{22} \\
a_{12} u_{21}-a_{11} u_{22} & a_{11} u_{22}^{2}-2 a_{12} u_{21} u_{22}+a_{22} u_{21}^{2}
\end{array}\right) .
\end{aligned}
$$

In the next section we shall need $Q_{11}$ and the explicit expression for this matrix element is

$$
Q_{11}(t)=\frac{2 \hbar}{\pi M^{2}} \int_{0}^{\infty} d \omega \operatorname{coth}\left(\frac{\beta \hbar \omega}{2}\right) J_{B}(\omega) \Delta(\omega, t)
$$

where $\Delta(\omega, t)$ is given by

$$
\Delta(\omega, t) \equiv \int_{0}^{t} d s \int_{0}^{t} d s^{\prime} g_{+}(t-s) \cos \omega\left(s-s^{\prime}\right) g_{+}\left(t-s^{\prime}\right)
$$

These results provide the tools needed to analyze the conditions under which passage to the quantum-classical limit may be carried out. In the next sections we present explicit results for both the Ohmic and super-Ohmic bath spectral densities.

\section{THERMALIZATION OF QUANTUM BROWNIAN MOTION}

Whether a linear or nonlinear system interacting with a bath will eventually reach thermal equilibrium or not is one of the fundamental issues in statistical mechanics. For a linear quantum brownian motion, from a general class of initial conditions, the state of $\mathrm{C}$ is considered to reach asymptotically the gaussian form 33 In this section, first we will show that the $\mathrm{C}$ subsystem evolution starting from an arbitrary initial condition possesses an asymptotic limit. We then study the influence of such a thermalized quantum system on A. For our analysis, we shall need the results for a system composed of the particle $C$ and the thermal bath $B$ in the absence of $A$. In this case eq. (36) takes the form,

$$
\begin{aligned}
\rho_{r W}\left(R_{t}, P_{t}, t\right)= & \int d R_{0} d P_{0} K_{C}\left(R_{t}, P_{t} ; t \mid R_{0}, P_{0} ; 0\right) \\
& \times \rho_{r W}\left(R_{0}, P_{0}, 0\right)
\end{aligned}
$$

In the absence of subsystem $A$, from eq. (38) the time evolution kernel for the Wigner function is

$$
K_{C}\left(R_{t}, P_{t} ; t \mid R_{0}, P_{0} ; 0\right)=N_{W}(t) \exp \left[-\delta \vec{X}_{W}^{T} \mathbf{Q}^{-1} \delta \vec{X}_{W}\right]
$$

where $\vec{X}_{W}^{T}=(R, P)$ and

$$
\langle\vec{X}\rangle=\left(\begin{array}{c}
\langle R\rangle \\
\langle P\rangle
\end{array}\right)=\frac{-1}{u_{21}}\left(\begin{array}{cc}
u_{11} & 1 \\
|\mathbf{u}| & u_{22}
\end{array}\right)\left(\begin{array}{c}
R_{0} \\
P_{0}
\end{array}\right) .
$$

For a harmonic potential, as a result of the Ehrenfest theorem, the center phase space coordinate $\langle\vec{X}\rangle$ of this distribution function follows a classical trajectory and will decay because of energy dissipation into the bath. Using the exact expressions for the matrix elements of $\mathbf{u}$, obtained from the formulas given in Appendix A, one may show that $\langle\vec{X}\rangle$ vanishes in the long time limit for both Ohmic and super-Ohmic baths. As a result, in both 
cases, for times long compared to the characteristic relaxation time for this decay, the time evolution kernel reduces to

$$
\begin{aligned}
K_{C}\left(R_{t}, P_{t} ; t \mid R_{0}, P_{0} ; 0\right) & \rightarrow N_{W}(t) \exp \left[-\vec{X}_{W}^{T} \mathbf{Q}^{-1} \vec{X}_{W}\right] \\
& \equiv K_{C}\left(R_{t}, P_{t} ; t\right)
\end{aligned}
$$

which is independent of $R_{0}$ and $P_{0}$. Consequently, eq. (44) reduces to

$$
\rho_{r W}\left(R_{t}, P_{t}, t\right)=K_{C}\left(R_{t}, P_{t} ; t\right) ;
$$

thus, for an arbitrary initial condition, the Wigner transformed reduced density matrix is uniquely determined by the time evolution kernel $K_{C}$. Furthermore, the offdiagonal elements of $\mathbf{Q}$ vanish in this limit and we obtain a Gaussian form for the density matrix whose widths are uniquely specified and given by $\left\langle(\Delta R)^{2}\right\rangle=Q_{11} / 2$ and $\left\langle(\Delta P)^{2}\right\rangle=Q_{22} / 2$. The width $\left\langle(\Delta R)^{2}(\infty)\right\rangle$ can be obtained from eq. (42) as

$$
\left\langle(\Delta R)^{2}(\infty)\right\rangle=\frac{\hbar}{\pi M^{2}} \int_{0}^{\infty} d \omega \operatorname{coth}\left(\frac{\beta \hbar \omega}{2}\right) J_{B}(\omega) \Delta(\omega, \infty),
$$

We show in Appendix A for the Ohmic case and for the weak coupling limit of the super-Ohmic case, that $J_{B}(\omega) \Delta(\omega, \infty)=M^{2} \chi_{C}^{\prime \prime}(\omega)$, where

$$
\chi_{C}^{\prime \prime}(\omega)=\frac{1}{M} \frac{\omega \gamma^{\prime}(\omega)}{\left(\omega_{r}^{2}-\omega^{2}+\omega \gamma^{\prime \prime}(\omega)\right)^{2}+\omega^{2} \gamma^{\prime 2}(\omega)},
$$

is the dynamical susceptibility for $C$. For the spectral density defined in eq. (5), the explicit form of the frequency dependent dissipation coefficient $\gamma(\omega)$ is given by

$$
M \gamma(\omega) \rightarrow \begin{cases}\eta & (\nu=1) \\ \eta \omega^{2} & (\nu=3)\end{cases}
$$

in the limit of a large cutoff parameter $\Lambda$, where $\gamma^{\prime \prime}(\omega)$ was absorbed in the mass renormalization for the $\nu=3$ case. Consequently, we may write $\left\langle(\Delta R)^{2}(\infty)\right\rangle$ as

$$
\left\langle\Delta^{2} R(\infty)\right\rangle=\frac{\hbar}{\pi} \int_{0}^{\infty} d \omega \operatorname{coth}\left(\frac{\beta \hbar \omega}{2}\right) \chi_{C}^{\prime \prime}(\omega) .
$$

Equation (52) is a statement of the fluctuationdissipation relation. 34 The form in eq. (52) suggests that the combined system $C B$ is now in thermal equilibrium with spectrum specified by $\chi_{C}^{\prime \prime}(\omega)$ but the system $C$ itself is not in equilibrium. The fluctuation-dissipation relation is violated at finite $t$ while the system is still far from equilibrium and holds only asymptotically. This result generalizes the result previously obtained by Caldeira and Leggett for an Ohmic bath.22

When the $C B$ system is in thermal equilibrium, we show in Appendix B using linear response theory that $J_{C B}(\omega)=\lambda^{2} \chi_{C}^{\prime \prime}(\omega)$ : the effective spectral density is proportional to the dynamical susceptibility of the $C$ system. If we use the relation

$$
J_{B}(\omega)=M \omega \gamma^{\prime}(\omega)
$$

for the bath spectral density assumed in eq. (5), we see that the effective spectral density in the combined system $C B$ can be written as

$$
J_{C B}(\omega)=\frac{\lambda^{2} J_{B}(\omega)}{M^{2}\left(\omega_{r}^{2}-\omega^{2}+\omega \gamma^{\prime \prime}(\omega)\right)^{2}+J_{B}^{2}(\omega)} .
$$

This relation can also be obtained by solving classical equations of motion as suggested earlier 6, 35, 36 owing to the linear coupling assumed between the $C$ system and the bath.

\section{EMERGENCE OF QUANTUM-CLASSICAL DYNAMICS VIA DECOHERENCE}

The investigation of the emergence of quantumclassical dynamics presented here will be restricted to cases where the dynamics of subsystem $A$ occurs on time scales which are very long compared to those that characterize subsystem $C$. We suppose that subsystem $A$ is a two-level system with $\sigma= \pm 1$ and focus on the extreme non-adiabatic regime where the population dynamics of subsystem $A$ is essentially frozen on the time scales of interest so that $\rho_{A}(-1,-1, t)=\rho_{A}(-1,-1,0), \rho_{A}(1,1, t)=$ $\rho_{A}(1,1,0)$. We further assume that subsystems $A$ and $C$ are decoupled initially and use the factorized initial condition,

$$
\hat{\rho}_{A C}\left(x_{0}, x_{0}^{\prime}, 0\right)=\hat{\rho}_{A}(0) \otimes \rho_{C}\left(x_{0}, x_{0}^{\prime}, 0\right) .
$$

This is a reasonable assumption if they are weakly coupled.

\section{A. Decoherence in $A$ interacting with an equilibrium $C B$ bath}

If the interaction between the $A$ system and the combined system $C B$ is turned on after an equilibrium state is reached, the effect of $C B$ on $A$ is given simply by the harmonic bath directly coupled to the $A$ subsystem but with its spectrum characterized by the effective spectral density. Under these conditions, the off-diagonal part of the density matrix can be obtained from eq. (13) by dropping the dependence on $\left(x, x^{\prime}\right)$, omitting the integrals over spin variables and by making the variable replacements $x \rightarrow \sigma$ and $J_{B} \rightarrow J_{C B}$ in eq. (18) for the influence functional. We find,

$$
\rho_{A}(-1,1, t)=e^{-D_{A}^{(0)}(t)} \rho_{A}(-1,1,0)
$$

where $D_{A}^{(0)}(t)$ is a decoherence factor for $\mathrm{A}$ and is defined as 


$$
\begin{aligned}
D_{A}^{(0)}(t)= & \frac{4}{\pi \hbar} \int_{0}^{\infty} d \omega J_{C B}(\omega) \operatorname{coth}\left(\frac{\beta \hbar \omega}{2}\right) \\
& \times\left(\frac{1-\cos (\omega t)}{\omega^{2}}\right) .
\end{aligned}
$$

Thus, as mentioned above, the dynamics of the composite system is equivalent to that of a two-level spin-boson system coupled to a harmonic bath, with an effective spectral density.
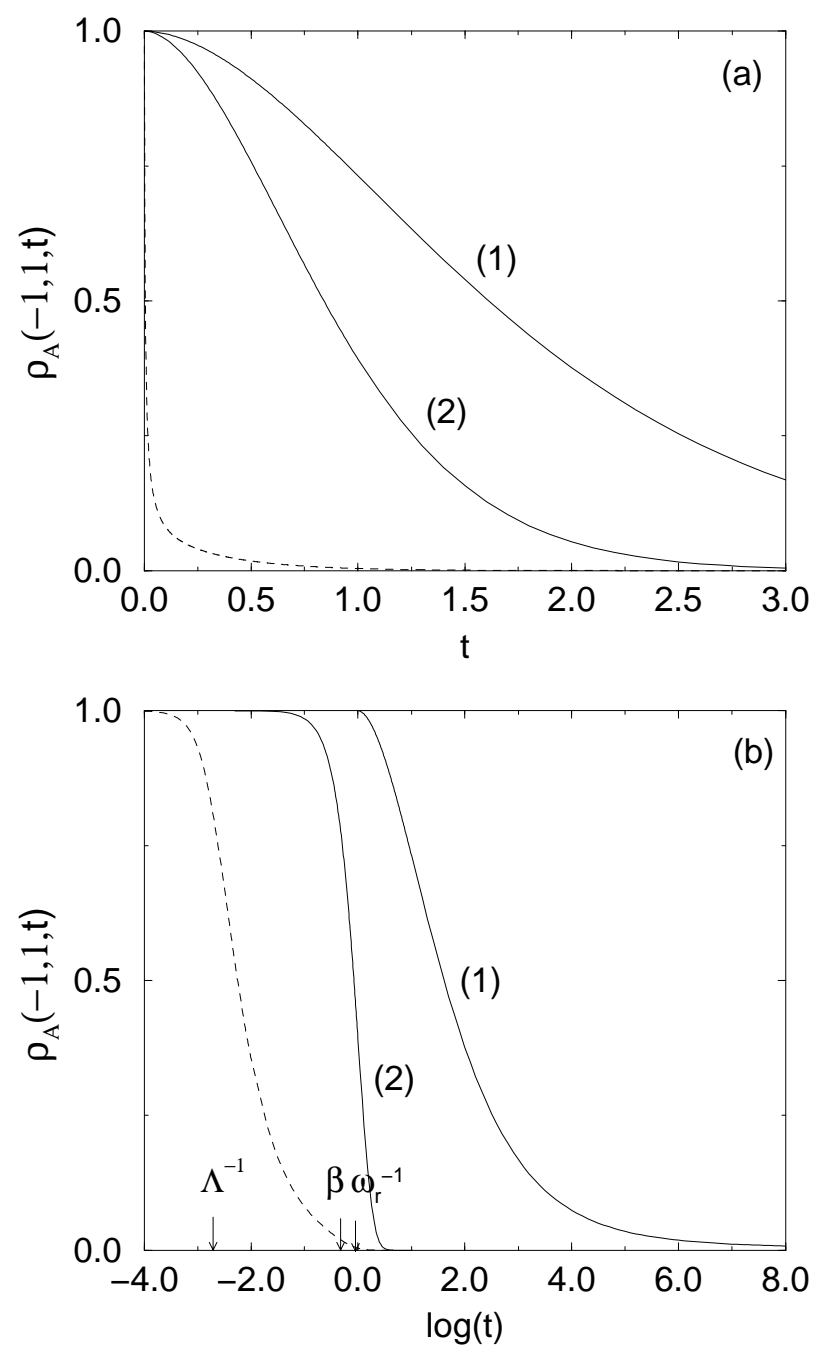

FIG. 1. The temporal behavior of the off-diagonal element of the reduced density matrix $\rho_{A}(-1,1, t)$ for subsystem $A$ is plotted in panel (a) versus time. The initial value $\rho_{A}(-1,1,0)=1$. Parameters are: $\beta=0.66, \Lambda=500, \omega_{r}=1$, $\gamma=0.3, M=1$ and $\hbar=1$. The same quantity is shown in panel (b) using a logarithmic time scale. In both figures the solid line corresponds to $A$ under the influence of $C B$ with the effective Ohmic spectral density, while the dashed line shows the influence of Ohmic bath $B$ without $C$. (1) $\lambda^{2}=0.1$ and (2) $\lambda^{2}=0.3$.

Using these results we may examine the coherence in $A$ under the influence of the effective bath $C B$ and the coherence of $A$ under the influence of the bath $B$ sepa- rately. In Figs. 1(a) and (b), the off-diagonal part of the density matrix of subsystem $A$ is plotted for an Ohmic environment.

The solid line corresponds to our system while the dashed line is the ordinary spin boson model without $C$. The time evolution of $\rho_{A}(-1,1, t)$ for the ordinary ordinary spin boson model is characterized by (1) an initial period where subsystem $A$ system has not yet felt the existence of the environment and its coherence is maintained; (2) a quantum regime for $t>1 / \Lambda$ where the system begins to interact with the vacuum fluctuations of the environment and, finally, (3) a thermal regime for $t>\hbar \beta$ where the effects of thermal fluctuations on subsystem $A$ have set in. For $1 / \Lambda<t<\hbar \beta$, only vacuum fluctuations interact with the system.

By contrast, the existence of the intermediate subsystem $C$ changes the evolution of $\rho_{A}(-1,1, t)$ significantly. Subsystem $C$ is characterized by its harmonic oscillator frequency and mass. Fluctuations originating in the bath $B$ are modulated by $C$ through these parameters. For a large mass $M$, the effective modes of the combined $C B$ system are concentrated in the neighborhood of $\omega_{r}$ and the decoherence behavior of subsystem $A$ is governed by these modes. Therefore, after the initial period, decoherence will begin to occur at $t \sim 1 / \omega_{r}$.

Although some systems are approximately characterized by an Ohmic spectral density, generic systems will have non-Ohmic spectral densities. The density of states typically varies as $\omega^{\nu}$ with $\nu$ depending on the spatial dimension $D$. We consider the $3 \mathrm{D}$ super-Ohmic case with $\nu=3$. Such an environment is relevant for the study of polarons 31 macroscopic magnetization tunneling in crystals 37 and radiation damping of atoms $38 \mathrm{~A}$ super-Ohmic environment affects subsystem $A$ evolution on short time scales more significantly than an Ohmic environment. A sub-Ohmic environment induces nontrivial long time behavior. Results for the super-Ohmic case with $\nu=3$ are shown in Figs. 2 (a) and (b).

For the super-Ohmic high dimensional environment, the vacuum fluctuations play a more significant role than for an Ohmic environment, reflecting the fact that there are larger number of high frequency modes in the higher dimensional environment. The same effect is also responsible for the large difference in the behavior of correlation functions of the system in quaptum and classical baths when the bath is super-Ohmic. 9 The dynamics of $A$ in the presence of the intermediate subsystem $C$ is worth noting. For the present choice of parameters, $\rho_{A}(-1,1, t)$ asymptotically reaches a non-zero value indicating that quantum coherence in the $A$ system will never be lost completely. The origin of this behavior can be understood in terms of the time-dependence of the diffusion coefficient (See Ref 26 ). System $C$ initially executes brownian motion with a time-dependent diffusion coefficient as a result of the non-Markovian time evolution of the reduced density matrix. This time-dependent diffusion coefficient of $C$, which also determines the decoherence rate, exhibits a rapid increase at early times $t>1 / \Lambda$ and 
then asymptotically vanishes. This initial increase can be large enough to wash away the quantum coherence of $C$ directly coupled to the bath. Owing to the modulation effect from $C$, these high frequency modes are filtered out and do not directly affect $A$ if $\omega_{r}<<\Lambda$. The late time value of the diffusion coefficient is too small to eliminate the quantum coherence from subsystem $A$ completely. Hence, the non-Markonvian nature of the density matrix evolution for the super-Ohmic case is responsible for the significant difference in the dynamics of coherence between the $A$ and $C$ subsystems.
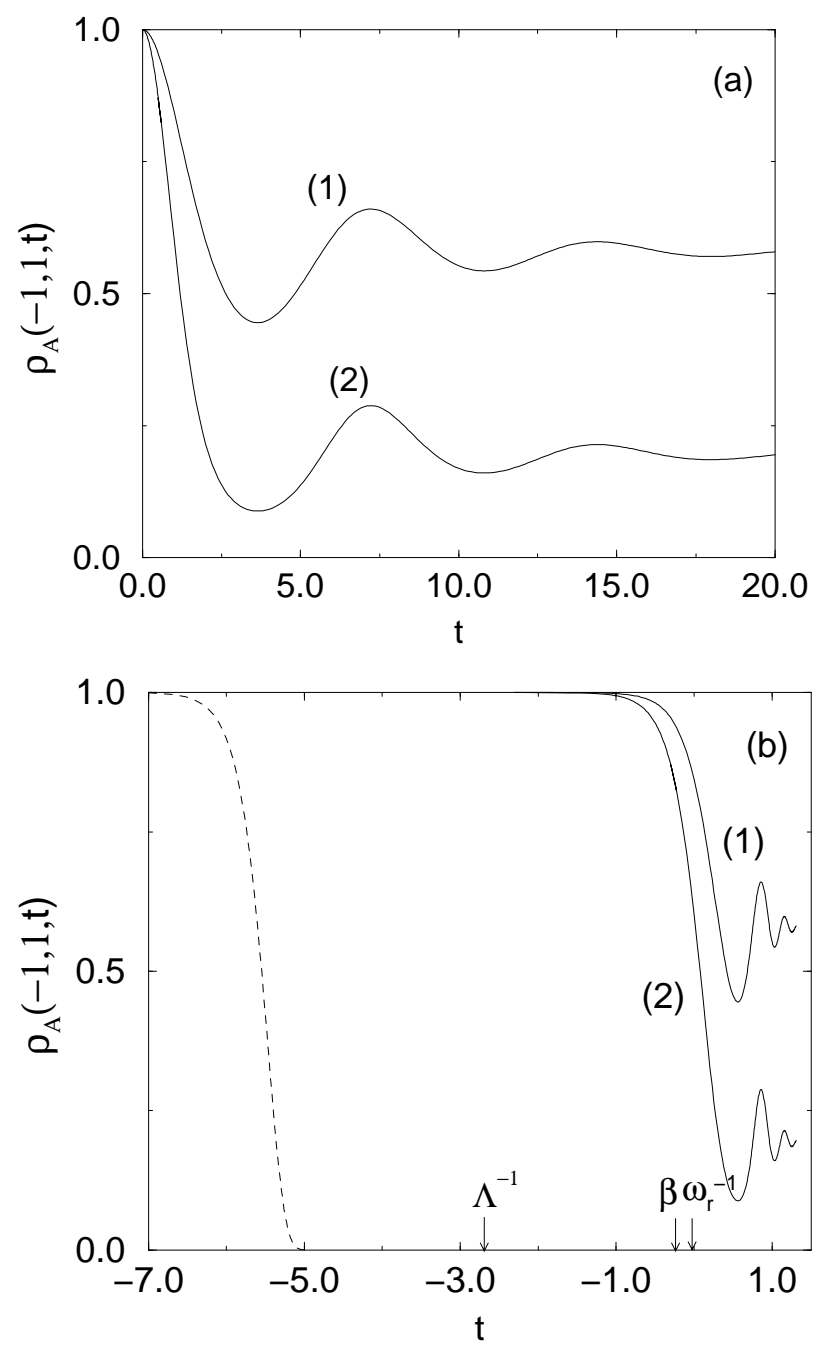

FIG. 2. Plot of $\rho_{A}(-1,1, t)$ versus time (a) and versus $\log (t)$ (b) for a super-Ohmic environment with $\nu=3$. Parameters and labeling are the same as in Fig. 1. The dashed line in panel (a) for super-Ohmic bath $B$ without $C$ overlaps the vertical axis. The behavior is visible in panel (b) using the logarithmic time scale.

\section{B. Decoherence in $A$ for arbitrary $C$ initial conditions}

Thus far we have considered only the asymptotic limit where $C B$ has reached equilibrium and the fluctuationdissipation relation holds. It is interesting to consider the coherence of $A$ under the influence of intrinsic dynamics of $C$ starting from arbitrary $C$ initial conditions. As an example of this situation, we assume that $C$ is initially in a gaussian coherent state given by the wave function,

$$
\psi_{\bar{x} \bar{p}}(x)=\frac{1}{\left(\pi \epsilon^{2}\right)^{1 / 4}} \exp \left[-\frac{(x-\bar{x})^{2}}{2 \epsilon^{2}}+i \frac{\bar{p} x}{\hbar}\right],
$$

with width $\epsilon=1 / \sqrt{\omega_{r}}$ where $\bar{x}$ and $\bar{p}$ are parameters. The $C$ subsystem density matrix at the initial time is then given by

$$
\rho_{C}\left(x_{0}, x_{0}^{\prime}, 0\right)=\frac{1}{\sqrt{\pi \epsilon^{2}}} \exp \left[-\frac{\left(R_{0}-\bar{x}\right)^{2}}{\epsilon^{2}}-\frac{r_{0}^{2}}{4 \epsilon^{2}}+i \frac{\bar{p} r_{0}}{\hbar}\right] \text {. }
$$

We may now use this expression for $\rho_{C}\left(x_{0}, x_{0}^{\prime}, 0\right)$ in the factorized initial condition for $A C$ given in eq. (55). The off-diagonal element of reduced density matrix for subsystem $A$ in the non-adiabatic limit can then be determined from eqs. (13) and (14) for $\rho_{r}\left(\sigma, \sigma^{\prime}, x, x^{\prime}, t\right)$ for $A C$ by integrating over the $C$ variables. The result is,

$$
\begin{aligned}
\rho_{A}(-1,1, t)= & \int d x d x^{\prime}\left[\int d x_{0} d x_{0}^{\prime}\right. \\
& \left.\times J_{C}^{-1,1}\left(x, x^{\prime}, t \mid x_{0}, x_{0}^{\prime}, 0\right) \rho_{C}\left(x_{0}, x_{0}^{\prime}, 0\right)\right] \\
& \times \rho_{A}(-1,1,0) .
\end{aligned}
$$

Changing integration variables $\left(x, x^{\prime}\right) \rightarrow(R, r)$ and $\left(x_{0}, x_{0}^{\prime}\right) \rightarrow\left(R_{0}, r_{0}\right)$ and using eq. (31) for $J_{C}^{-1,1}\left(R, r, t \mid R_{0}, r_{0}, 0\right)$ we may write this as

$$
\begin{aligned}
\rho_{A}(-1,1, t)= & \int d R d r\left[\int d R_{0} d r_{0}\right. \\
& \left.\times N(t) \exp \left\{\frac{i}{\hbar} \mathcal{L}\right\} \rho_{C}\left(R_{0}, r_{0}, 0\right)\right] \\
& \times \rho_{A}(-1,1,0) .
\end{aligned}
$$

Making use of the expression for $\mathcal{L}$ evaluated for $\sigma=-1$ and $\sigma^{\prime}=1$ and carrying out the integrations we find,

$$
\begin{aligned}
\rho_{A}(-1,1, t)= & \rho_{A}(-1,1,0) e^{-D_{A}(t)} \\
& \times \exp \left[\frac{i}{\hbar}\left(f_{R}(t) \bar{x}+f_{P}(t) \bar{p}\right)\right] \\
& \times \exp \left[-\frac{1}{\hbar}\left(f_{R}^{2}(t) / M \omega_{r}+f_{P}^{2}(t) M \omega_{r}\right)\right],
\end{aligned}
$$

where

$$
f_{R}(t)=\lambda \int_{0}^{t} d s \frac{u_{11}(s)}{u_{21}(s)}, \quad f_{P}(t)=\lambda \int_{0}^{t} d s \frac{1}{u_{21}(s)}
$$

and 


$$
D_{A}(t)=\frac{2 \lambda^{2}}{\pi \hbar M^{2}} \int_{0}^{\infty} d \omega J_{B}(\omega) \operatorname{coth}\left(\frac{\beta \hbar \omega}{2}\right) \Delta_{A}(\omega, t)
$$

The explicit expression for $\Delta_{A}(\omega, t)$ can be computed from the solutions presented in Appendix A by a lengthy but straightforward calculation. We find,

$$
\begin{aligned}
& \Delta_{A}(\omega, t)=\frac{1}{\left(\Omega_{r}^{2}-\omega^{2}\right)^{2}+4 \Gamma^{2} \omega^{2}}\left[2 \frac{1-\cos (\omega t)}{\omega^{2}}\right. \\
& \quad+\frac{2}{\Omega \Omega_{r}^{2}} \frac{1-\cos (\omega t)}{\omega} \\
& \quad \times\left(\omega \Omega\left(1-e^{-\Gamma t} \cos (\Omega t)\right)-\omega \Gamma e^{-\Gamma t} \sin (\Omega t)\right) \\
& \quad+\frac{2}{\Omega \Omega_{r}^{2}} \frac{\sin (\omega t)}{\omega} \\
& \quad \times\left(2 \Gamma \Omega\left(1-e^{-\Gamma t} \cos (\Omega t)\right)+\left(\Omega^{2}-\Gamma^{2}\right) e^{-\Gamma t} \sin (\Omega t)\right) \\
& +\frac{1}{\Omega^{2} \Omega_{r}^{4}}\left\{\left(\omega^{2}+4 \Gamma^{2}\right) \Omega^{2}\left(1-\cos (\Omega t) e^{-\Gamma t}\right)^{2}\right. \\
& \quad-2 \Gamma \Omega\left(\omega^{2}+2 \Gamma^{2}-2 \Omega^{2}\right) \\
& \quad \times\left(1-\cos (\Omega t) e^{-\Gamma t}\right) \sin (\Omega t) e^{-\Gamma t} \\
& \left.\left.+\left(\omega^{2} \Gamma^{2}+\left(\Omega^{2}-\Gamma^{2}\right)^{2}\right) \sin ^{2}(\Omega t) e^{-2 \Gamma t}\right\}\right]
\end{aligned}
$$

Equations (62)-(65) are our main results. The first term in eq. (62) is the initial condition for $A$, the second term accounts for the decoherence arising from thermal fluctuations of the bath mediated by $C$. The third term arises from the initial condition for $C$ and the last term is responsible for the decoherence of $A$ due to the averaged damped oscillatory motion of $C$.

The modulus of the off-diagonal reduced density matrix element for $A$ is shown in Figs. 3(a) and (b) as a function of time. The solid line shows the second term eq. (62), $S \equiv e^{-D_{A}(t)}$, which gives the decoherence due to the quantum-back-reaction-induced self-interaction of subsystem $A$. The dashed line shows the last term, $L \equiv \exp \left[-\frac{1}{\hbar}\left(f_{R}^{2}(t) / M \omega_{r}+f_{P}^{2}(t) M \omega_{r}\right)\right]$, the decoherence due to the motion of $C$. The latter effect is not strong enough to eliminate coherence of $A$ completely. Since, initially, the $\mathrm{CB}$ is far from equilibrium, those modes that affect $\mathrm{A}$ are not necessarily near resonant modes around $\omega_{r}$. Consequently, the decoherence of A is more rapid in this case than in the case of evolution from the thermal equilibrium initial condition studied in Sec. IV. A. This tendency is more evident in a superOhmic bath than in an Ohmic bath. With generic initial conditions for $C, \mathrm{~A}$ is under the influence of the nonequilibrium bath $C B$, which is no longer equivalent to the effective thermal bath with the spectral density $J_{C B}$.
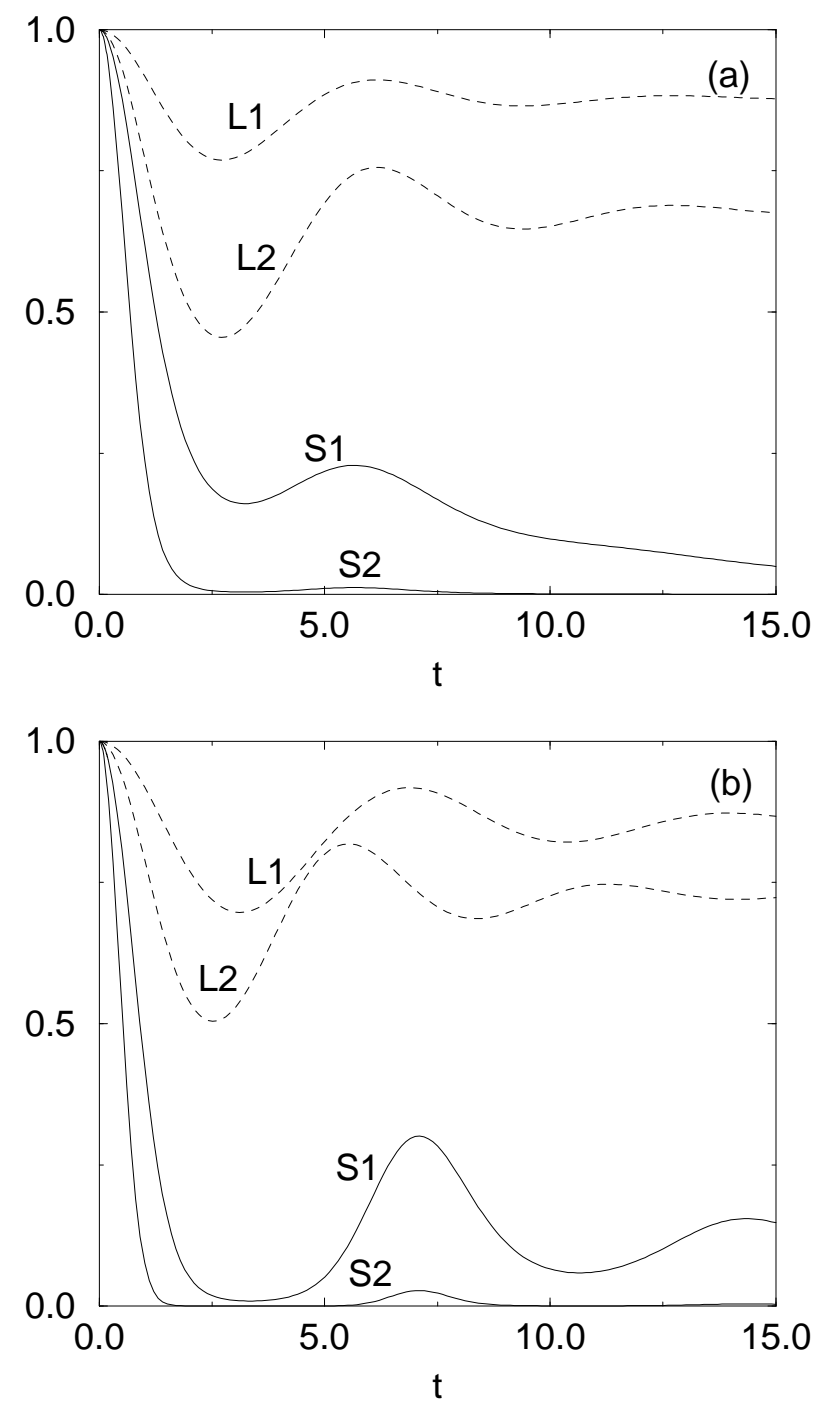

FIG. 3. Plot of contribution to the modulus of $\rho_{A}(-1,1, t)$ versus time for an Ohmic bath (Fig. 3(a)) and for a super Ohmic bath (Fig. B(b)). Subsystem $C$ is in a coherent state initially. In both figures the solid line corresponds to the second term $\mathrm{S}$, while the dashed line corresponds to the last term $\mathrm{L}$ in the right hand side in eq.(62). $\lambda^{2}=0.1$ for S1,L1 and $\lambda^{2}=0.3$ for S2,L2. Parameters values are the same as in Fig. 1.

Now let us consider the situation where the decoherence of $C$ is fast and that of $A$ is slow. This may occur at high temperatures and for weak coupling between $A$ and $C$. In such a case, the back reaction from $A$ on $C$ may be neglected. In this circumstance, we may consider the decoherence of $C$ in the absence of $A$. Quantum brownian motion and decoherence of a damped harmonic oscillator has been studied mreviously.22.24 27 Using the result in eq. (3.9) of Ref. 26 the reduced density matrix may be approximated by,

$$
\rho_{r}\left(\sigma, \sigma^{\prime}, x, x^{\prime}, t\right) \sim e^{-\left(x-x^{\prime}\right)^{2} D_{C}(t)} \rho_{r}\left(\sigma, \sigma^{\prime}, x, x^{\prime}, 0\right),
$$

where $D_{C}(t)=\int_{0}^{t} d s \int_{0}^{s} d s^{\prime} \alpha^{R}\left(s^{\prime}\right) \cos \omega_{r} s^{\prime}$ is the decoher- 
ence factor for $C$.
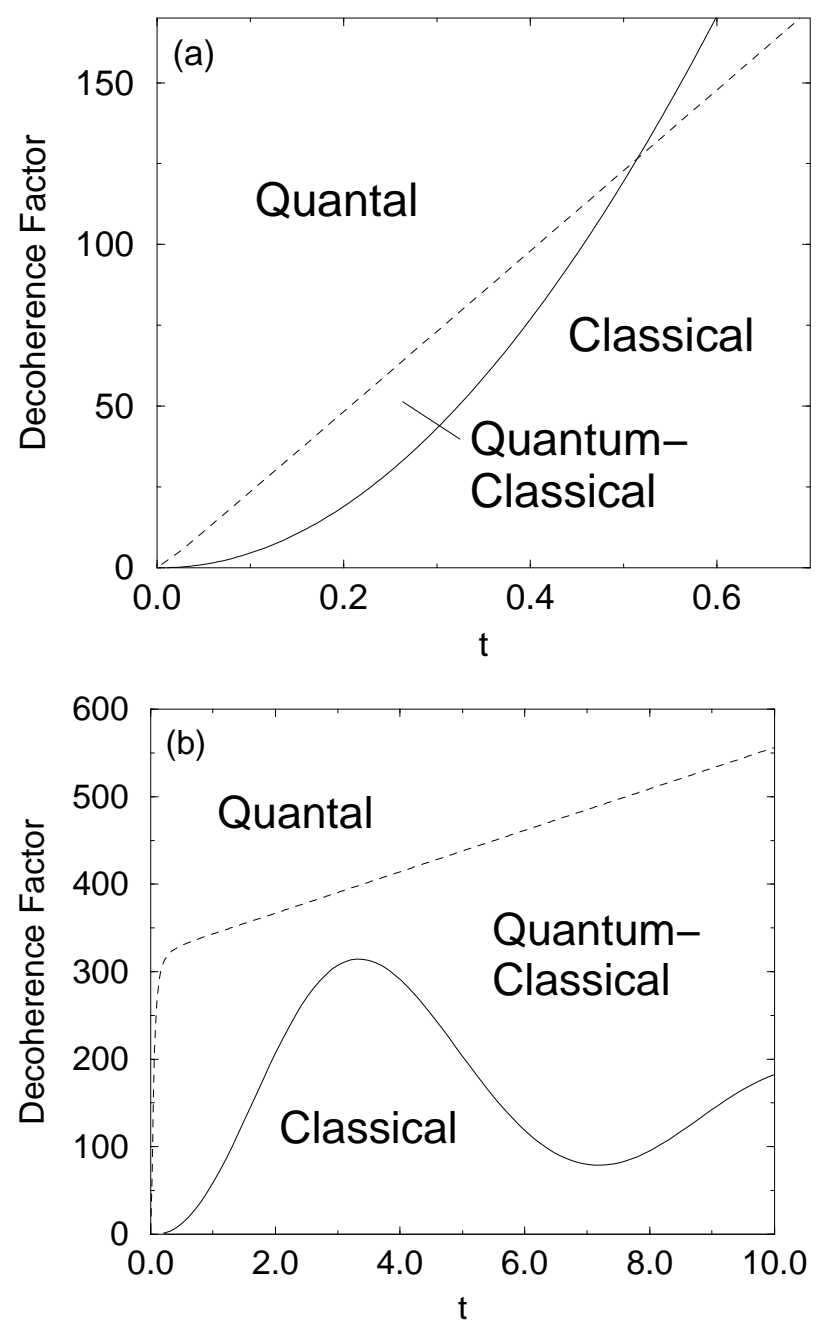

FIG. 4. Plot of the decoherence factor versus time. The solid line corresponds to the logarithm of the modulus of the density matrix $\rho_{A}$ in eq.(62) with the inverse sign, while the dashed line is $D_{C}$. System parameters are: $x-x^{\prime}=1, \omega_{r}=1$, $\gamma=0.3, M=1, \hbar=1$. Panel (a) is for the Ohmic bath with $\beta=0.002, \Lambda=500$. Panel (b) is for the $\nu=3$ super-Ohmic bath with $\beta=0.02, \Lambda=20$.

In Fig. 1 , the decoherence factors for $A$ and $C$ are plotted. For an Ohmic bath (Fig. 目(a)), initially, the quantum coherence is lost faster $C$ than in $A$; hence, the $A$ subsystem behaves more quantum mechanically than the $C$ subsystem during this initial period. Figure $4(\mathrm{a})$ shows that there is a crossover and for longer times, $t>1 / \omega_{r}$, subsystem $A$ experiences stronger decoherence than $C$. The decoherence factor for $C$ varies linearly with $t$ indicating that the dynamics of $C$ asymptotically approaches Markovian evolution in the high temperature Ohmic bath. For a super-Ohmic bath (Fig. ్(b)), a substantial portion of the quantum coherence in $C$ is lost during the initial period due to large fluctuations coming from the bath, while $A$ retains quantum coherence for a long time. The dynamics of the coupled system can be approximated by mixed quantum-classical evolution for long times after the short transient period.

\section{CONCLUSION}

An appreciation of the conditions under which a quantum mechanical system may be approximated as a quantum-classical system is essential in many applications to the dynamics of many-body systems. Adopting an open quantum system point of view has provided a natural way to explore this issue based on the decoherence of the superposition of quantum states into a statistical mixture under the influence of the environment. 22,29 Indeed, considerations of decoherence have played an important role in discussions of schemes for the simulation of condensed phase systems 39

Using influence functional methods, we have shown for our simple model system that the decoherence time scales that characterize the $A$ and $C$ subsystems can differ significantly. In particular, in the limit of nonadiabatic dynamics we have identified the following three regimes: (1) the full quantum regime where both the $A$ and $C$ subsystems behave quantum mechanically; (2) the quantumclassical regime where subsystem $A$ maintains coherence owing to its indirect coupling to the bath, while $C$ has lost its coherence and behaves effectively classically; (3) the classical regime where the quantum coherence of both the $A$ and $C$ subsystems has been lost and the composite $A C$ subsystem exhibits effectively classical dynamics. The different roles that the environment plays in its effect on two subsystems is responsible for the separation of decoherence time scales.

We also saw that a different choice of initial conditions will modify above picture. When the initial condition for $C$ is chosen to be a pure gaussian coherent state, $C B$ can no longer be considered to be a thermal bath and the coherence of $A$ will be lost in a shorter time scale than times given by the inverse characteristic frequency of $C$.

Quantum/classical correspondence in nonlinear systems, in particular, in chaotic systems, has many nontrivial features 41 The quantum open system approach adopted in this paper can also shed light on this problem 42,0 While the model system studied here is very simple and the extreme non-adiabatic regime is only one limiting case to consider, our results nevertheless have provided some insight into the emergence of quantumclassical dynamics and should be useful in the study of the quantum dynamics of more complex systems.

\section{ACKNOWLEDGMENTS}

This work was supported in part by a grant from the Natural Sciences and Engineering Research Council of 
Canada. Acknowledgment is made to the donors of The Petroleum Research Fund, administered by the ACS, for partial support of this research.

\section{APPENDIX A: SOLUTIONS OF EQUATIONS OF MOTION}

In this Appendix we give some details of the solutions of the equations of motion discussed in Sec. IIC which are used in the calculations presented in Sec. IV. We require the functions $u_{1,2}(s)$ (and $v_{1,2}(s)$ ) which are the solutions of the homogeneous parts of the Euler-Lagrange equations (22) and (23). These functions can be found if $g_{ \pm}(s)$, which are also solutions of the homogeneous parts eqs. (22) and (23), are known, since

$$
u_{1}(s)=\dot{g}_{+}(s)-\frac{g_{+}(s)}{g_{+}(t)} \dot{g}_{+}(t), \quad u_{2}(s)=\frac{g_{+}(s)}{g_{+}(t)},
$$

in order to satisfy the boundary conditions on these functions. Using the spectral density for an Ohmic bath, the equation that $g_{ \pm}(s)$ satisfies is

$$
\ddot{g}_{ \pm}(s) \pm 2 \Gamma \dot{g}_{ \pm}(s)+\omega_{r}^{2} g_{ \pm}(s)=0
$$

The solution of eq. (A2) is

$$
g_{ \pm}(s)=\frac{\sin \Omega s}{\Omega} e^{\mp \Gamma s}
$$

where $\Gamma=\gamma$ and $\Omega^{2} \equiv \omega_{r}^{2}-\gamma^{2}$.

Using the spectral density for a super-Ohmic bath with $\nu=3$, the equations of motion for $g_{ \pm}(s)$ can be written as

$$
\ddot{g}_{ \pm}(s)+\omega_{r}^{2} g_{ \pm}(s) \mp 2 \gamma \dddot{g}_{ \pm}(s)=0
$$

Thus, the equation of motion has the form of the Abraham-Lorentz equation.40 This equation is an approximation to the full equation of motion with non-local time dependence. As in the electromagnetic field case, only the physically relevant roots of the characteristic equation must be retained. These are $\Gamma \pm i \Omega$ with

$$
\Gamma \equiv \frac{1}{6 \gamma}\left(\frac{D}{4}+\frac{1}{D}-1\right), \quad \Omega \equiv \frac{\sqrt{3}}{6 \gamma}\left(\frac{D}{4}-\frac{1}{D}\right)
$$

where

$$
D=2\left(1+54\left(\gamma \omega_{r}\right)^{2}+\sqrt{108} \gamma \omega_{r} \sqrt{27\left(\gamma \omega_{r}\right)^{2}+1}\right)^{1 / 3}
$$

Retaining the physically relevant roots of the characteristic equation, the solution for $g_{ \pm}(s)$ for the super-Ohmic case has the same form as eq. A3 but with the above values of $\Gamma$ and $\Omega$. In the weak coupling limit that we consider in our calculations, $\Gamma \rightarrow \gamma \omega_{r}^{2}$ and $\Omega \rightarrow \omega_{r}^{2}$.

In our model, eq. (A4) can be reduced to a second order ordinary differential equation by differentiating the homogeneous part of the Euler-Lagrange equations with respect to time as

$$
\dddot{g}_{ \pm}(s)=-\omega_{r}^{2} \dot{g}_{ \pm}(s)+\mathcal{O}(\gamma)
$$

which may be substituted into eq. A4 to give (A2) with $\Gamma=\gamma \omega_{r}^{2}$ and $\Omega=\omega_{r}$ in the leading order approximation as indicated above. Although one can improve the approximation leading to eq. A2 for the super-Ohmic case to arbitrary higher order in $\gamma$, we restrict our study to the leading order in this parameter.

Since the $u_{1,2}$ and $v_{1,2}$ solutions are now known, we have all the information needed to compute the various quantities necessary to obtain the numerical results. In particular, the expression for the matrix elements of $\mathbf{a}$ in eq. (28) for a general environment can be found. (See Refs.22,26.27 for an Ohmic bath.) In our computations, we need $a_{11}(t)$ which has the form,

$$
a_{11}(t)=\frac{1}{2 \pi g_{+}^{2}(t)} \int_{0}^{\infty} d \omega J_{B}(\omega) \operatorname{coth} \frac{\beta \hbar \omega}{2} \Delta(\omega, t),
$$

where $\Delta(\omega, t)$ was defined in eq. (43). The evaluation of $\Delta(\omega, t)$ is straightforward and leads to,

$$
\begin{aligned}
& \Delta(\omega, t)=\frac{1}{2 \Omega^{2}} \frac{1}{\left(\Omega_{r}^{2}-\omega^{2}\right)^{2}+4 \Gamma^{2} \omega^{2}}\left[2 \Omega^{2}+e^{-\Gamma t}\right. \\
& \quad \times\left[2 \cos (\omega t)\left\{\left(\Gamma^{2}+\omega_{+} \omega_{-}\right) \cos (\Omega t)-2 \Gamma \Omega \sin (\Omega t)\right\}\right. \\
& \left.-\left(\Gamma^{2}+\omega_{-}^{2}\right) \cos \left(\omega_{+} t\right)-\left(\Gamma^{2}+\omega_{+}^{2}\right) \cos \left(\omega_{-} t\right)\right] \\
& +e^{-2 \Gamma t}\left\{\Omega_{r}^{2}+\omega^{2}+2 \Gamma \Omega \sin (2 \Omega t)\right. \\
& \left.\left.-\left(\Gamma^{2}+\omega_{+} \omega_{-}\right) \cos (2 \Omega t)\right\}\right]
\end{aligned}
$$

where $\omega_{ \pm} \equiv \omega \pm \Omega$ and $\Omega_{r}^{2} \equiv \Omega^{2}+\Gamma^{2}$. Using this result, in the asymptotic limit we obtain,

$$
J_{B}(\omega) \Delta(\omega, \infty)= \begin{cases}\frac{2 M \gamma \omega}{\left(\Omega_{r}^{2}-\omega^{2}\right)^{2}+4 \gamma^{2} \omega^{2}} & (\nu=1) \\ \frac{2 M \gamma \omega^{3}}{\left(\Omega_{r}^{2}-\omega^{2}\right)^{2}+4 \gamma^{2} \omega^{6}} & (\nu=3) .\end{cases}
$$

In writing this equation, we used the fact that the distribution $J_{B}(\omega) \Delta(\omega, \infty)$ is highly peaked around $\omega_{r}$ for small $\gamma$.

Similarly, for long times we may write the elements of a as,

$$
\begin{gathered}
a_{11}(t) \rightarrow \frac{\Omega^{2} e^{2 \Gamma t}}{2 \pi \sin ^{2} \Omega t} \int_{0}^{\infty} d \omega J_{B}(\omega) \operatorname{coth} \frac{\beta \hbar \omega}{2} \Delta(\omega, \infty) \\
a_{12}(t)=a_{21}(t) \rightarrow \frac{\Omega e^{\Gamma t}}{2 \pi \sin \Omega t} \int_{0}^{\infty} d \omega J_{B}(\omega) \operatorname{coth} \frac{\beta \hbar \omega}{2} \\
(\Gamma-\Omega \cot (\Omega s)) \Delta(\omega, \infty) \\
a_{22}(t) \rightarrow \frac{1}{2 \pi} \int_{0}^{\infty} d \omega J_{B}(\omega) \operatorname{coth} \frac{\beta \hbar \omega}{2} \\
\left\{\omega^{2}+(\Gamma-\Omega \cot (\Omega s))^{2}\right\} \Delta(\omega, \infty) .
\end{gathered}
$$

Using these results we can compute the asymptotic properties of $\mathbf{Q}$ needed in the calculations presented in the text. 


\section{APPENDIX B: EFFECTIVE SPECTRAL DENSITY}

In this Appendix we derive the relation between the effective spectral density for the $C B$ system and the dynamical susceptibility of subsystem $C$ using an argument that by-passes the actual diagopalization procedure. A similar argument is given in Refs 35,36 From the Hamiltonian given in eq. (11), we can write the equation of motion for $C$ with a harmonic potential in the form,

$$
x(\omega)=\chi_{C}(\omega) F_{C}(\omega)
$$

in the complex Fourier representation with a susceptibility function $\chi_{C}(\omega)$. The force $F_{C}(\omega)=-\partial V_{A C}(\omega) / \partial x=$ $-\lambda \sigma(\omega)$ is the external force from subsystem $A$ acting on $C$. On the other hand, the equation of motion for subsystem $A$ gives

$$
-m_{A} \omega^{2} \sigma(\omega)-\lambda x(\omega)=F_{A}(\omega)
$$

with $F_{A}(\omega)=-\partial V_{A}(\sigma) / \partial \sigma$. Here we assumed $H_{A}$ has the form $p_{A}^{2} / 2 m_{A}+V_{A}(\sigma)$ for simplicity. Our argument does not depend on the form of $H_{A}$ as clear from the context. Combining with eq. (B1), we have

$$
\left[-m_{A} \omega^{2}-\lambda^{2} \chi_{C}(\omega)\right] \sigma(\omega)=F_{A}(\omega) .
$$

Now, suppose we have already diagonalized last three terms of eq. (1) and replaced them with $N+1$ - harmonic oscillators,

$H_{C}+V_{C B}+H_{B} \rightarrow H_{B}^{\prime}=\sum_{n=0}^{N}\left(\frac{p_{n}^{\prime 2}}{2 m_{n}^{\prime}}+\frac{m_{n}^{\prime} \omega_{n}^{\prime 2} q_{n}^{\prime 2}}{2}\right)$

We write the interaction term $V_{A C}$ in terms of new coordinates $q_{n}^{\prime}$

$$
V_{A C} \rightarrow V_{A B}^{\prime}=\sigma \sum_{n=0}^{N} c_{n}^{\prime} q_{n}^{\prime}
$$

Assuming that the system described by the Hamiltonian in eq. (11) reaches thermal equilibrium in the asymptotic limit, we can replace the effect of the combined system $C B$ by the equivalent thermal bath $H_{B}^{\prime}$ at the same temperature. Although this diagonalization procedure is straightforward, for our purpose, we only need the form of the equation of motion for $\sigma$ expressed by the dissipation coefficient $\gamma_{C B}(\omega)$ for the effective bath eq. (B4),

$$
\left[-m_{A} \omega^{2}-i m_{A} \omega \gamma_{C B}(\omega)\right] \sigma(\omega)=F_{A}(\omega) \text {. }
$$

Comparing the above with eq. (B3) we have

$$
\lambda^{2} \chi_{C}(\omega)=i m_{A} \omega \gamma_{C B}(\omega) .
$$

Recall that the spectral density is related to the dissipation coefficient by

$$
J_{C B}(\omega)=m_{A} \omega \gamma_{C B}^{\prime}(\omega)
$$

for real $\omega$. From this the desired relation follows:

$$
J_{C B}(\omega)=\lambda^{2} \chi_{C}^{\prime \prime}(\omega)
$$

[1] See, e.g., E. B. Davis, Quantum Theory of Open Systems, (Academic Press, London, 1976); B. J. Lindenberg and B. J. West, The Nonequilibrium Statistical Mechanics of Open and Closed Systems, (VCH Press, New York, 1990); T. Dittrich, P. Hänggi G.-L. Ingold, B. Kramer G. Schön and W. Zwerger, Quantum Transport and Dissipation, (Wiley, New York, 1998).

[2] S. Mukamel, Principles of Nonlinear Optical Spectroscopy, (Oxford University Press, New York, 1995).

[3] U. Weiss, Quantum Dissipative Systems, (World Scientific, Sigapore, 1999).

[4] S. Nakajima, Prog. Theor. Phys. 20, 948 (1958).

[5] R. Zwanzig, Lect. Theor. Phys. 3, 106 (1961).

[6] R. P. Feynman and F. L. Vernon, Ann. Phys. 24, 118 (1963).

[7] P. Pechukas and U. Weiss, eds., Special Issue, Quantum Dynamics of Open Systems, Chem. Phys, 268, (2001).

[8] D. E. Makarov and N. Makri, Chem. Phys. Lett. 221, 482 (1994); N. Makri and D. E. Makarov, J. Chem. Phys. 102, 4600, 4611 (1995); N. Makri, J. Math. Phys. 36, 2430 (1995); E. Sim and N. Makri, Comp. Phys. Commun. 99, 335 (1997): N. Makri, J. Phys. Chem. 102, 4414 (1998).

[9] S. A. Egorov, K. F. Everitt, and J. L. Skinner, J. Phys. Chem. A 103, 9494 (1999).

[10] V. May and O. Kühn, Charge and Energy Transfer Dynamics in Molecular Systems (Wiley, New York, 2000).

[11] J. C. Tully in Modern Methods for Multidimensional Dynamics Computations in Chemistry, ed. D. L. Thompson, (World Scientific, NY, 1998), p. 34.

[12] M. F. Herman, Annu. Rev. Phys. Chem. 45, 83 (1994); M. F. Herman, J. Chem. Phys. 87, 4779 (1987).

[13] J. C. Tully, J. Chem. Phys. 93, 1061 (1990); J. C. Tully, Int. J. Quantum Chem. 25, 299 (1991); D. S. Sholl and J. C. Tully, J. Chem. Phys. 109, 7702 (1998).

[14] L. Xiao and D. F. Coker, J. Chem. Phys. 100, 8646 (1994); D. F. Coker and L. Xiao, J. Chem. Phys. 102, 496 (1995); H. S. Mei and D.F. Coker, J. Chem. Phys. 104, 4755 (1996).

[15] F. Webster, P. J. Rossky, and P. A. Friesner, Comp. Phys. Comm. 63, 494 (1991); F. Webster, E. T. Wang, P. J. Rossky, and P. A. Friesner, J. Chem. Phys. 100, 483 (1994).

[16] T. J. Martinez, M. Ben-Nun, and R. D. Levine, J. Phys. Chem. A 101, 6389 (1997).

[17] O. V. Prezhdo and V. V. Kisil, Phys. Rev. A 56, 162 (1997).

[18] C. C. Martens and J.-Y. Fang, J. Chem. Phys. 106, 4918 
(1996); A. Donoso and C. C. Martens, J. Phys. Chem. 102, 4291 (1998).

[19] R. Kapral and G. Ciccotti, J. Chem. Phys. 110, 8919 (1999); S. Nielsen, R. Kapral and G. Ciccotti, J. Chem. Phys. 112, 6543 (2000); S. Nielsen, R. Kapral and G. Ciccotti, J. Stat. Phys. 101, 225 (2000); S. Nielsen, R. Kapral and G. Ciccotti, J. Chem. Phys. 115, 5805 (2001); D. Mac Kernan, G. Ciccotti and R. Kapral, J. Chem. Phys. 116, 2346 (2002).

[20] C. Wan and J. Schofield, J. Chem. Phys. 113, 7047 (2000).

[21] R. Kapral, J. Phys. Chem. 105, 2885 (2001).

[22] A. O. Caldeira and A. J. Leggett,Physica A121, 587 (1983);

[23] V. Hakim and V. Ambegaokar, Phys. Rev. A 32, 423 (1985).

[24] H. Grabert, P.Schramn and G. L. Ingold, Phys. Rep. 168, 115 (1988).

[25] W. G. Unruh and W. H. Zurek, Phys. Rev. D40, 1071 (1989);

[26] B. L. Hu, J. P. Paz and Y. Zhang, Phys. Rev. D 45, 2843 (1992).

[27] W. H. Zurek, S. Habib, and J. P. Paz, Phys. Rev. Lett. 70, 1187 (1993); B. L. Hu and Y. Zhang, Int. J. Mod. Phys. A10 (1995) 4537; C. Anastopoulos and J. J. Halliwell, Phys. Rev. D 51, 6870 (1995).

[28] W. H. Zurek, Phys. Rev. D24, 1516 (1981); D26, 1862 (1982); E. Joos and H. D. Zeh, Z. Phys. B59, 223 (1985).

[29] Decoherence and the Apperance of the Classical World in Quantum Theory, eds. D. Giulini, et al., (Springer, Berlin, 1996).

[30] C. Itzykson and J.-B. Zuber, Quantum Field Theory,
(McGraw Hill, New York, 1980).

[31] A. J. Leggett, S. Chakravarty, A. T. Dorsey, M. P. A. Fisher, A. Garg, and W. Zwerger, Rev. Mod. Phys. 59, 1 (1987).

[32] E. Wigner, Phys. Rev. 40, 749 (1932); K. Imre, E. Özizmir, M. Rosenbaum and P. F. Zwiefel, J. Math. Phys. 5, 1097 (1967).

[33] M. A. Huerta and H. S. Robertson, Jour. Stat. Phys. 1, 393 (1969); M. Tegmark and H. S. Shapiro, Phys. Rev. E 50, 2538 (1994).

[34] M. Toda, N. Saito, R. Kubo, and H. Hashizume Statistical Physics I,II (Springer, Berlin, 1991).

[35] A. J. Leggett, Phys. Rev. B30, 1208 (1984).

[36] A. Garg, J. N. Onuchic, and V. Ambegaokar, J. Chem. Phys. 83, 4491 (1985).

[37] A. Garg and G. H. Kim, Phys. Rev. Lett. 63, 2512 (1989).

[38] P. M. V. B. Barone and A. O. Caldeira, Phys. Rev. A 43, 57 (1991).

[39] E. R. Bittner and P. J. Rossky, J. Chem. Phys. 103, 8130 (1995); O. V. Prezhdo and P. J. Rossky, Phys. Rev. Lett. 81, 5294 (1998).

[40] J. D. Jackson, Classical Electrodynamics, (Wiley, New York, 1975).

[41] B. V. Chirikov, in Chaos and Quantum Physics Les Houches Lectures Session LII, eds M. J. Giannoni, A. Voros, and J. Zinn-Justin (North Holland 1991).

[42] K. Shiokawa and B. L. Hu, Phys. Rev. E52, 2497 (1995); S. Habib, K. Shizume, and W. H. Zurek, Phys. Rev. Lett. 80, 4361 (1998).

[43] J. P. Paz and W. H. Zurek, in Coherent Matter Waves, Les Houches Lectures Session LII, (North Holland, Amsterdam, 1999). 